\title{
Interaction analysis on transmissibility of main pathogens of hand, foot, and mouth disease in Xi'an: a modelling study
}

\section{Yao Bai}

The Fourth Military Medical University

Kun Liu

The Fourth Military Medical University Department of Epidemiology

Zhongjun Shao ( 13759981783@163.com )

https://orcid.org/0000-0002-3236-3629

\section{Zhijun Chen}

Xi'an Center for Disease Control and Prevention

Research article

Keywords: hand foot and mouth disease, interaction analysis, transmissibility

Posted Date: August 25th, 2020

DOI: https://doi.org/10.21203/rs.2.18494/v2

License: (a) (i) This work is licensed under a Creative Commons Attribution 4.0 International License.

Read Full License 
Title:

Interaction analysis on transmissibility of main pathogens of hand, foot, and mouth disease in Xi'an: a modelling study

Running head:

Interaction of main pathogens of hand, foot, and mouth disease

\section{Authors and affiliations:}

Yao Bai ${ }^{12^{2}}$, Kun Liu ${ }^{1}$, Zhongjun Shao ${ }^{1}$, Zhijun Chen $^{2}$

* These authors contributed equally to this study

1. Department of Epidemiology, School of Military Preventive Medicine, The Fourth Military Medical University, Xi'an, Shaanxi Province, People's Republic of China;

2. Department of Infection Disease Control and Prevention, Xi'an Center for Disease Prevention and Control, Xi'an, Shaanxi Province, People's Republic of China;

\# Corresponding author

Zhongjun Shao

Department of Epidemiology, School of Military Preventive Medicine, The Fourth Military Medical University

710032, 169 Changle Xilu Road, Xincheng District, Xi'an, Shaanxi Province, People's Republic of China

Tel: $+86-13759981783$ 


\begin{abstract}
Objectives: Hand, foot and mouth disease (HFMD) is a new infectious disease that has attracted much attention at home and abroad, and it spreads widely. In this study, we aimed to explore the interaction between the main pathogens of HFMD through the model of transmission dynamics.
\end{abstract}

Method: The database of HFMD in Xi'an City was established and the main pathogens (EV71, CoxA16, other enteroviruses) were monitored for a long time. In the study, we defined the ascending period of reported cases as $R_{a s c}$, and the descending period as $R_{d e s}$, and calculated the regeneration number of the two periods using susceptible-infectious-recovered model.

Results: In Xi'an City, $R_{a s c}$ and $R_{\text {des }}$ of HFMD was 1.39 (95\% CI: $\left.1.31-1.48\right)$ and 0.70 (95\% CI: 0.65 0.74), respectively. CoxA16 and other enteroviruses interacted with each other and decreased the value of $R_{\text {asc }}$. However, $R_{\text {des }}$ of CoxA16 was increased by other enteroviruses directionally. However, during the reported cases decreasing period, interactions only occurred between EV71 and other enteroviruses and between CoxA16 and other enteroviruses. These interactions all decreased $R_{\text {asc }}$ but increased $R_{\text {des }}$ of affected pathogens.

Conclusions: There is interaction among pathogens of HFMD in Xi'an City. The reproduction number of pathogens increased or decreased to a certain extent after interaction, and finally approached 1.

\title{
INTRODUCTION
}


Hand, foot and mouth disease (HFMD), as an emerging infectious disease, has long been regarded as a major challenge to the health care system. Since it was first discovered in New Zealand in 1957, HFMD has continued to break out in Malaysia, Shanghai, Singapore, Japan, Australia and other countries and regions, and has become an important infectious disease concerned by countries all over the world[1-4]. Previous studies have shown that the transmissibility of HFMD can be calculated by susceptible-infectious-removed model[5]. The basic reproduction rate basic reproduction number $\left(R_{0}\right)$ is usually used as a threshold parameter to predict whether infection will spread or not. It is defined as the expected number of secondary infections caused by an individual during the entire infection period in a susceptible population[6]. The previous research on the transmission dynamic model of HFMD has been relatively complete[7-12], including the study on the transmissibility of the main pathogens of HFMD[13]. Based on this, our study further explored the interaction of transmissibility among the main pathogens of HFMD, so as to reveal the deep transmission mechanism of HFMD pathogens.

In this study, the data of reported cases of HFMD in Xi'an City were collected and updated, and longterm etiological surveillance was carried out to calculate the incidence of major pathogens of HFMD. In the study, the SIR model was used to calculate the transmissibility of the three pathogens and found their interaction.

\section{METHODS}

\section{Study sites and data collection}

In this study, Xi'an City in Shaanxi Province were selected as study sites. Xi'an City is the capital of Shaanxi 
Province, the vice-provincial city, the core city of Guanzhong Plain(Central Shaanxi Plain) urban agglomeration, an important central city in western China approved by the State Council, and an important national scientific research, education and industrial base (Figure 1).

Based on the reported cases and deaths in Xi'an City from 2009 to 2016, a database was established. Five fecal or anal swab samples from clinically diagnosed cases in various districts and counties were collected on time and sent to the laboratory of the Municipal Centers for Disease Control and Prevention. (Enteroviruses 71 [EV71], Coxsackieviruses A16 [CoxA16] and other enteroviruses, referred to as "other") were detected by polymerase chain reaction (PCR). We counted the proportion of these three major pathogens to estimate the number of people infected with these three pathogens in HFMD cases. In addition, population of the cities, birth rate and death rate of the population were collected from statistical yearbooks announced by the statistical bureau of the two cities.

\section{Patient and public involvement}

The patients and the public were not involved in this study.

\section{Simulation model and transmissibility estimation}

A susceptible-infectious-recovered (SIR) model was adopted considering the natural birth rate and death rate of the population. The equations of the model were shown as follows:

$$
\begin{aligned}
& \frac{d S}{d t}=a N-\beta S I-b S \\
& \frac{d I}{d t}=\beta S I-(\gamma+b+f) I \\
& \frac{d R}{d t}=\gamma I-b R
\end{aligned}
$$


$N=S+I+R$

The model divides the population into four parts, namely $S, I, R$ and $N$, representing susceptible individuals, infected individuals, recovered individuals and the whole population. Parameters $\beta$ and $\gamma$ refer to the relative transmission rate and recovery rate of HFMD. Parameters $a, b$ and $f$ represent birth rate, death rate and fatality.

The transmission rate of HFMD is quantified by the reproduction number. In the calculation, we define the ascending period of reported cases as $R_{a s c}$, and the descending period as $R_{d e s}$. The calculation formula is as follows:

$R_{a s c}=\frac{\beta_{a s c} S_{0}}{\gamma+b+f}$

$R_{\text {des }}=\frac{\beta_{d e s} S_{0}}{\gamma+b+f}$

Some studies have found differences in pathogenicity of HFMD pathogens, and our study speculate that there may also be differences in infectivity among different pathogens[14]. In the study, if there is a difference between the transmissibility calculated when two or more pathogens spread at the same time and that calculated by a single pathogen, it is considered that there is an interaction between pathogens. The mathematical model was used to fit the transmissibility of pathogens in different scenes, and on this basis, statistical methods were used to test whether the difference is statistically significant.

\section{Parameter estimation and statistical analysis}

Parameters $a, b$, and $f$ were calculated from the collected data. According to the published study [7, 11], the infectious period of HFMD was about two weeks, therefore $\gamma=0.5$. From the collected data of reported HFMD cases, an epidemic peak was divided into ascending and descending parts which were employed to 
fit the SIR model to calculate $\beta_{a s c}$ and $\beta_{\text {des }}$ in each year.

Berkeley Madonna 8.3.18 (developed by Robert Macey and George Oster of the University of California at Berkeley. Copyright (C1993-2001 Robert I. Macey \& George F. Oster) was employed to run the model and least root mean square was adopted to assess goodness of fit. SPSS 13.0 (IBM Corp., Armonk, NY, USA) was employed to analyze the difference and the interaction of $\beta_{a s c}$ and $\beta_{\text {des }}$ among the three main pathogens of the disease. Eleven models (Linear, Logarithmic, Inverse, Quadratic, Cubic, Power, Compound, S, Logistic, Growth, Exponential, and Logistic) in SPSS 13.0 were employed to fit the yearly trends of reported HFMD cases and fatality. The 11 curve models and equations are as follows ("Y" represents $R_{0}$, " $x$ " represents the time [year]):

Linear regression (LIN): $Y=b_{0}+b_{1} x$

Logarithmic curve (LOG): $Y=b_{0}+b_{1} \ln (x)$

Inverse function curve (INV): $Y=b_{0}+b_{1} / x$

Quadratic curve (QUA): $Y=b_{0}+b_{1} x+b_{2} x^{2}$

Cubic curve (CUB): $Y=b_{0}+b_{1} x+b_{2} x^{2}+b_{3} x^{3}$

Composite curve (COM): $Y=b_{0}+b_{1}{ }^{x}$

Power function curve (POW): $Y=b_{0}+x^{b_{1}}$

S-shaped curve (S):Y $=e^{\left(b_{0}+b_{1} / x\right)}$

Growth curve (GRO): $Y=e^{\left(b_{0}+b_{1} x\right)}$

Logistic curve (LGS): $Y=1 /\left(1 / \mu+b_{0} b_{1}{ }^{x}\right)$ 
Exponential curve (EXP): $Y=b_{0} e^{b_{1} x}$

\section{RESULTS}

\section{Epidemiological features of the disease in Xi'an City}

A total of 170567 cases of HFMD were reported in Xi'an City from January 2009 to December 2016, of which 30 cases died and the case fatality rate was $0.02 \%$. According to the fitting results of the linear model, the number of reported cases of HFMD showed an upward trend year by year, and the equation was " $Y=$ $14661.57+1479.845 X^{\prime \prime}$, where $X$ represented the number of reported cases and $Y$ represented the order of years, and the results were not statistically significant $\left(R^{2}=0.326, F=2.896, P=0.140\right)$ (Figure 2$)$. According to the fitting results of the inverse model, the fatality rate of the disease decreased year by year, and the equation was " $Y=0.007+0.034 / X$ ", where $Y$ and $X$ represented the fatality rate and the order of years, respectively, which was statistically significant $\left(R^{2}=0.727, F=15.958, P=0.007\right)$ (Figure 3). From 2009 to $2016,7,710$ stool or anal swab specimens were collected from the reported cases, among which, 2,496 were tested EV71 positive, 1,434 were Cox A16 positive, and 1,434 were other enteroviruses positive.

\section{Transmissibility of the three pathogens}

The results of model fitting showed that 18 epidemic peaks of HFMD cases were reported from 2009 to 2016 in Xi'an City, with $R_{a s c}$ and $R_{\text {des }}$ of $1.39(95 \% C I: 1.31-1.48)$ and 0.70 (95\% CI: $\left.0.65-0.74\right)$, respectively (Figure 4-a). The number of epidemic peaks of EV71, CoxA16, and other enterovirus was 16, 18, and 17, respectively. The $R_{a s c}$ of EV71, CoxA16, and other enterovirus was 1.47 (95\% CI: 1.37 - 1.57) (Figure 4-b), $1.61(95 \%$ CI: $1.34-1.88)$ (Figure 4-c), and 1.50 (95\% CI: 1.38 - 1.61) (Figure 4-d), with no statistical 
significance among the three pathogens $(F=0.626, P=0.539)$. The $R_{\text {des }}$ of EV71, CoxA16, and other enterovirus was 0.64 (95\% CI: 0.58 - 0.70) (Figure 4-b), 0.57 (95\% CI: 0.48 - 0.67) (Figure 4-c), and 0.66 $(95 \%$ CI: $0.60-0.71)$ (Figure 4-d), with no statistical significance among the three pathogens $(F=1.506, P$ $=0.232$ ). During the period of the study, the transmissibility of HFMD in Xi'an City of basically remained stable and fluctuated. In 2010-2011, the transmissibility of CoxA16 fluctuated briefly (Figure 5).

\section{Interaction of the three pathogens}

To analyze the interaction of the three pathogens, we compared the transmissibility for each other among the three pathogens (Table 1). We found that difference of $R_{\text {asc }}$ between CoxA16 (mean $\left.=1.61\right)$ and CoxA16+Other $($ mean $=1.25)$ and the difference between Other $($ mean $=1.50)$ and CoxA16+Other $($ mean $=$ 1.25) were statistically significant in Xi'an City. There was no statistical significance of $R_{\text {asc }}$ between any other two groups in the city. There was also no statistical significance of $R_{\text {des }}$ between any two groups in the city except between CoxA16 $($ mean $=0.57)$ and CoxA16+Other $($ mean $=0.70)($ Table 1$)$. These differences revealed that, during the reported cases increasing period, CoxA16 and other enteroviruses interacted with each other and their transmissibility $\left(R_{a s c}\right)$ was weaken by the interaction (Figure 6-a). However, during the reported cases decreasing period, the interaction of the two viruses was directional, and the transmissibility $\left(R_{\text {des }}\right)$ of CoxA16 increased when other enteroviruses spread in the same period (Figure 6-b).

\section{DISCUSSION}

Observing the changing trend of HFMD cases in Xi'an City, we found that the number of reported cases increased year by year, while the fatality rate decreased year by year. The increase in the number of reported cases indicates that pathogens exist for a long time and spread widely in Xi'an City. The downward trend of case fatality indicates that the health and epidemic prevention departments may have taken relevant 
prevention and control measures at this stage and the hospital's ability to diagnose and report HFMD may have increased.

Since the decline period of reported cases may include the effective phase of interventions, the assessment of the transmissibility of HFMD focuses on its rising period, that is, the value of $R_{\text {asc }}$. The results showed that the $R_{\text {asc }}$ of EV71, CoxA16 and other enteroviruses were 1.47, 1.61, 1.50 respectively. According to the existing research, the reproduction number of influenza is $1.4-2.0$ [15]. After comparison, it can be considered that the transmissibility of HFMD is lower than that of influenza. Therefore, our research results are more in line with the reality. From the model fitting results, we can find that the transmissibility of HFMD pathogens is generally stable, so the spread and epidemic of HFMD in Xi'an City cannot be stopped in a short time. Based on this, the surveillance, prevention and control measures for HFMD should not be relaxed, and the relevant institutions should strengthen the publicity and education among the population, so as to make them pay attention to personal hygiene and reduce aggregation activities.

The results of interaction analysis showed that CoxA16 and other enteroviruses interacted with each other in Xi'an City during the reported cases increasing period. The transmissibility of CoxA16 or other enteroviruses might be decreased if the two viruses co-transmit in the same population. However, during the reported cases decreasing period, the interaction was directional.

The reason for this pathogen interaction in Xi'an City remains unclear. It might result from the geographic difference. Xi'an City locates in the central west of China, which has warm and semi-humidy continental monsoon climate (temperature, rainfall, and relative humidity), as well as developed socioeconomic factors [16-19]. These factors might affect the activity of the pathogens and the mode of contact among individuals. Furthermore, it is unknown whether the pathogens have the special genomic 
features in Xi'an City.

Our study also found the results of the interaction between pathogens, and the transmissibility of pathogens was enhanced and weakened in varying degrees after the interaction. The potential causes of this phenomenon need to be further explored. In the next step, we will also carry out investigation and research on this phenomenon, in order to provide more evidence for the control and prevention of HFMD.

Contributors: $\mathrm{YB}$ and $\mathrm{KL}$ designed the study. $\mathrm{YB}, \mathrm{ZC}, \mathrm{KL}$ and $\mathrm{ZS}$ collected data. $\mathrm{YB}$ and $\mathrm{ZC}$ and performed the analysis. YB wrote the first draft of this paper. All authors contributed to the writing of the manuscript.

Funding: This project was supported by the Shaanxi Provincial Health and Family Planning Research Foundation (2016A002).

Competing interests: None declared.

Data sharing statement: Extra data is available by emailing to the first authors (Yao Bai, 402359346@qq.com) on reasonable request.

Acknowledgments: The authors thank all study participants for providing the data and field investigators for collecting the data.

\section{References}

1. Chan KP, Goh KT, Chong CY, Teo ES, Lau G, Ling AE: Epidemic hand, foot and mouth disease caused by human enterovirus 71, Singapore. Emerging infectious diseases 2003, 9(1):78-85.

2. Chan LG, Parashar UD, Lye MS, Ong FG, Zaki SR, Alexander JP, Ho KK, Han LL, Pallansch MA, Suleiman AB et al: Deaths of children during an outbreak of hand, foot, and mouth disease in sarawak, malaysia: clinical and pathological characteristics of the disease. For the Outbreak Study Group. Clinical infectious diseases : an official publication of the Infectious Diseases Society of America 2000, 31(3):678- 
683.

3. Fujimoto T, Chikahira M, Yoshida S, Ebira H, Hasegawa A, Totsuka A, Nishio O: Outbreak of central nervous system disease associated with hand, foot, and mouth disease in Japan during the summer of 2000: detection and molecular epidemiology of enterovirus 71. Microbiology and immunology 2002, 46(9):621-627.

4. Xu M, Su L, Cao L, Zhong H, Dong N, Xu J: Enterovirus genotypes causing hand foot and mouth disease in Shanghai, China: a molecular epidemiological analysis. BMC infectious diseases 2013, 13:489.

5. Chen S, Yang D, Liu R, Zhao J, Yang K, Chen T: Estimating the transmissibility of hand, foot, and mouth disease by a dynamic model. Public health 2019, 174:42-48.

6. Heffernan JM, Smith RJ, Wahl LM: Perspectives on the basic reproductive ratio. Journal of the Royal Society, Interface 2005, 2(4):281-293.

7. Du Z, Zhang W, Zhang D, Yu S, Hao Y: Estimating the basic reproduction rate of HFMD using the time series SIR model in Guangdong, China. PloS one 2017, 12(7):e0179623.

8. Lai CC, Jiang DS, Wu HM, Chen HH: A dynamic model for the outbreaks of hand, foot, and mouth disease in Taiwan. Epidemiology and infection 2016, 144(7):1500-1511.

9. Li Y, Zhang J, Zhang X: Modeling and preventive measures of hand, foot and mouth disease (HFMD) in China. International journal of environmental research and public health 2014, 11(3):3108-3117.

10. Wang Y, Feng Z, Yang Y, Self S, Gao Y, Longini IM, Wakefield J, Zhang J, Wang L, Chen X et al: Hand, foot, and mouth disease in China: patterns of spread and transmissibility. Epidemiology 2011, 22(6):781792.

11. Takahashi S, Liao Q, Van Boeckel TP, Xing W, Sun J, Hsiao VY, Metcalf CJ, Chang Z, Liu F, Zhang J et al: Hand, Foot, and Mouth Disease in China: Modeling Epidemic Dynamics of Enterovirus Serotypes and Implications for Vaccination. PLoS medicine 2016, 13(2):e1001958.

12. Wang J, Xiao Y, Cheke RA: Modelling the effects of contaminated environments on HFMD infections in mainland China. Bio Systems 2016, 140:1-7.

13. Ma E, Fung C, Yip SH, Wong C, Chuang SK, Tsang T: Estimation of the basic reproduction number of enterovirus 71 and coxsackievirus $\mathrm{A} 16$ in hand, foot, and mouth disease outbreaks. The Pediatric infectious disease journal 2011, 30(8):675-679.

14. Xing W, Liao Q, Viboud C, Zhang J, Sun J, Wu JT, Chang Z, Liu F, Fang VJ, Zheng Y et al: Hand, foot, and mouth disease in China, 2008-12: an epidemiological study. The Lancet Infectious diseases 2014, 14(4):308-318

15. Ferguson NM, Cummings DA, Fraser C, Cajka JC, Cooley PC, Burke DS: Strategies for mitigating an influenza pandemic. Nature 2006, 442(7101):448-452. 
16. Li L, Qiu W, Xu C, Wang J: A spatiotemporal mixed model to assess the influence of environmental and socioeconomic factors on the incidence of hand, foot and mouth disease. BMC public health 2018, 18(1).

17. Gou F, Liu X, He J, Liu D, Cheng Y, Liu H, Yang X, Wei K, Zheng Y, Jiang X et al: Different responses of weather factors on hand, foot and mouth disease in three different climate areas of Gansu, China. BMC infectious diseases 2018, 18(1):15.

18. Yang $\mathrm{H}$, Wu J, Cheng J, Wang $\mathrm{X}$, Wen L, Li K, Su H: Is high relative humidity associated with childhood hand, foot, and mouth disease in rural and urban areas? Public health 2017, 142:201-207.

19. Liao J, Yu S, Yang F, Yang M, Hu Y, Zhang J: Short-Term Effects of Climatic Variables on Hand, Foot, and Mouth Disease in Mainland China, 2008-2013: A Multilevel Spatial Poisson Regression Model Accounting for Overdispersion. PloS one 2016, 11(1):e0147054. 


\section{Figure legends}

Figure 1. Geographical Location of Xi'an City in China.

Figure 2. Yearly number of reported HFMD cases and its trend in Xi'an City.

Figure 3. Fatality of HFMD and its trend in Xi'an City.

Figure 4. Results of model fitting and the transmissibility of HFMD's three pathogens. a: EV71+CA16+Other; b: EV71: c, CA16; d: Other; e: EV71+CA16; f: EV71 +Other; g: CA16+Other.

Figure 5. The trends of the transmissibility of HFMD. a: $R_{a s c} ; \mathrm{b}: R_{d e s}$.

Figure 6. The interaction modes of the transmissibility of HFMD. a: $R_{a s c} ; \mathrm{b}: R_{d e s}$; Arrow indicates the direction of interaction from one pathogen to another one; no arrow refers to no interaction. 


\section{Tables}

Table 1. Interaction of transmissibility of HFMD's three pathogens.

\begin{tabular}{llcc}
\hline Transmissibility & Compared groups & $t$ & $p$ \\
\hline$R_{\text {asc }}$ & EV71 v.s. EV71+CA16 & 0.831 & 0.413 \\
& EV71 v.s. EV71+Other & 0.300 & 0.766 \\
& EV71 v.s. EV71+CA16+Other & 1.110 & 0.275 \\
& CA16 v.s. CA16+EV71 & 1.306 & 0.201 \\
& CA16 v.s. CA16+Other & 2.206 & 0.034 \\
CA16 v.s. CA16+EV71+Other & 1.489 & 0.146 \\
Other v.s. Other+EV71 & 0.615 & 0.543 \\
Other v.s. Other+CA16 & 2.151 & 0.038 \\
\hline Other v.s. Other+EV71+CA16 & 1.388 & 0.174 \\
\hline EV71 v.s. EV71+CA16 & -0.982 & 0.334 \\
\hline$R_{\text {des }}$ & EV71 v.s. EV71+Other & & 0.348 \\
\hline
\end{tabular}




\begin{tabular}{lcc}
\hline EV71 v.s. EV71+CA16+Other & -1.625 & 0.114 \\
$\begin{array}{l}\text { CA16 v.s. CA16+EV71 } \\
\text { CA16 v.s. CA16+Other }\end{array}$ & -1.857 & 0.073 \\
$\begin{array}{l}\text { CA16 v.s. CA16+EV71+Other } \\
\text { Other v.s. Other+EV71 }\end{array}$ & -0.614 & 0.543 \\
Other v.s. Other+CA16 & -2.375 & 0.023 \\
Other v.s. Other+EV71+CA16 & -0.483 & 0.632 \\
\hline
\end{tabular}


Figures

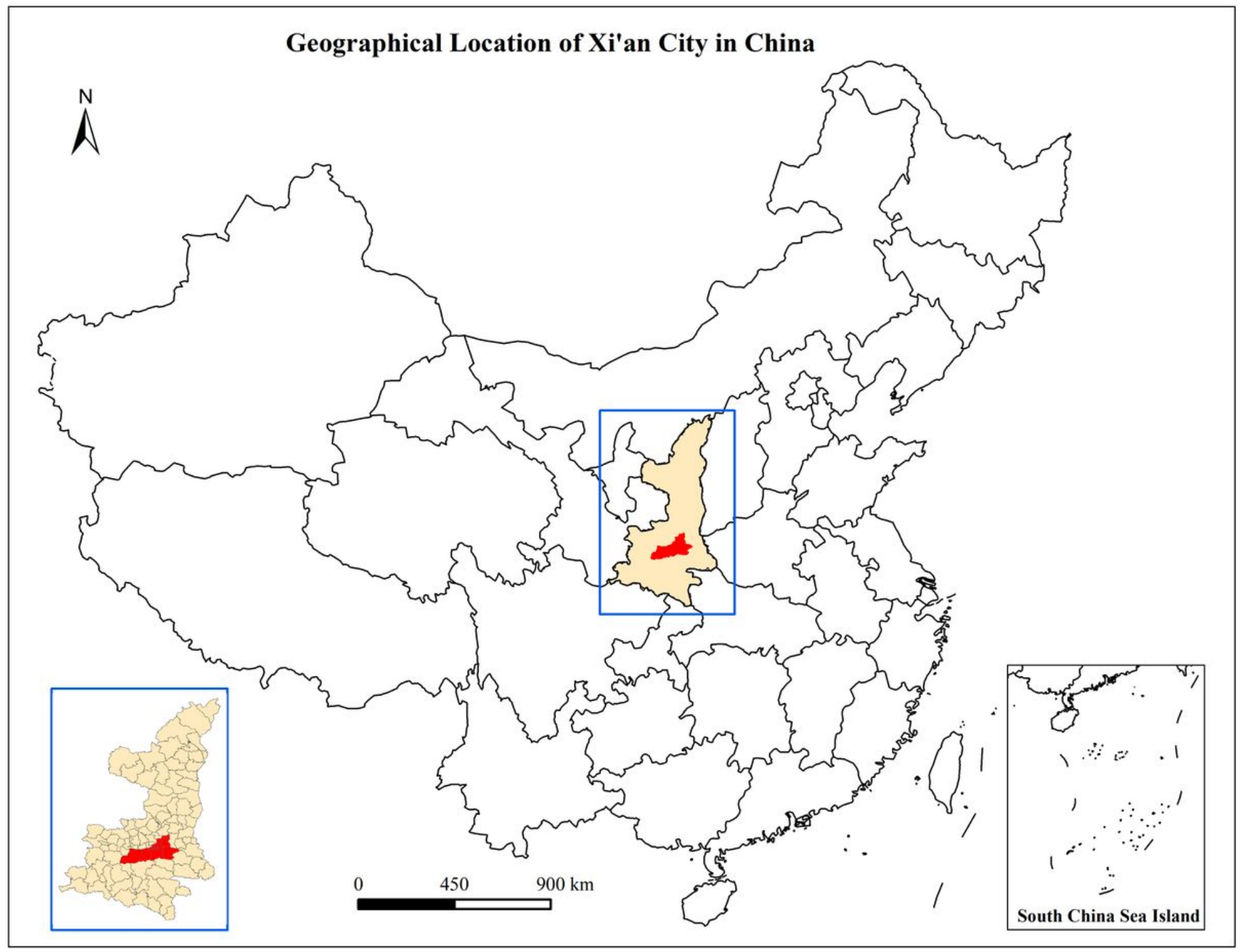

Figure 1

Geographical Location of Xi'an City in China. Note: The designations employed and the presentation of the material on this map do not imply the expression of any opinion whatsoever on the part of Research Square concerning the legal status of any country, territory, city or area or of its authorities, or concerning the delimitation of its frontiers or boundaries. This map has been provided by the authors. 


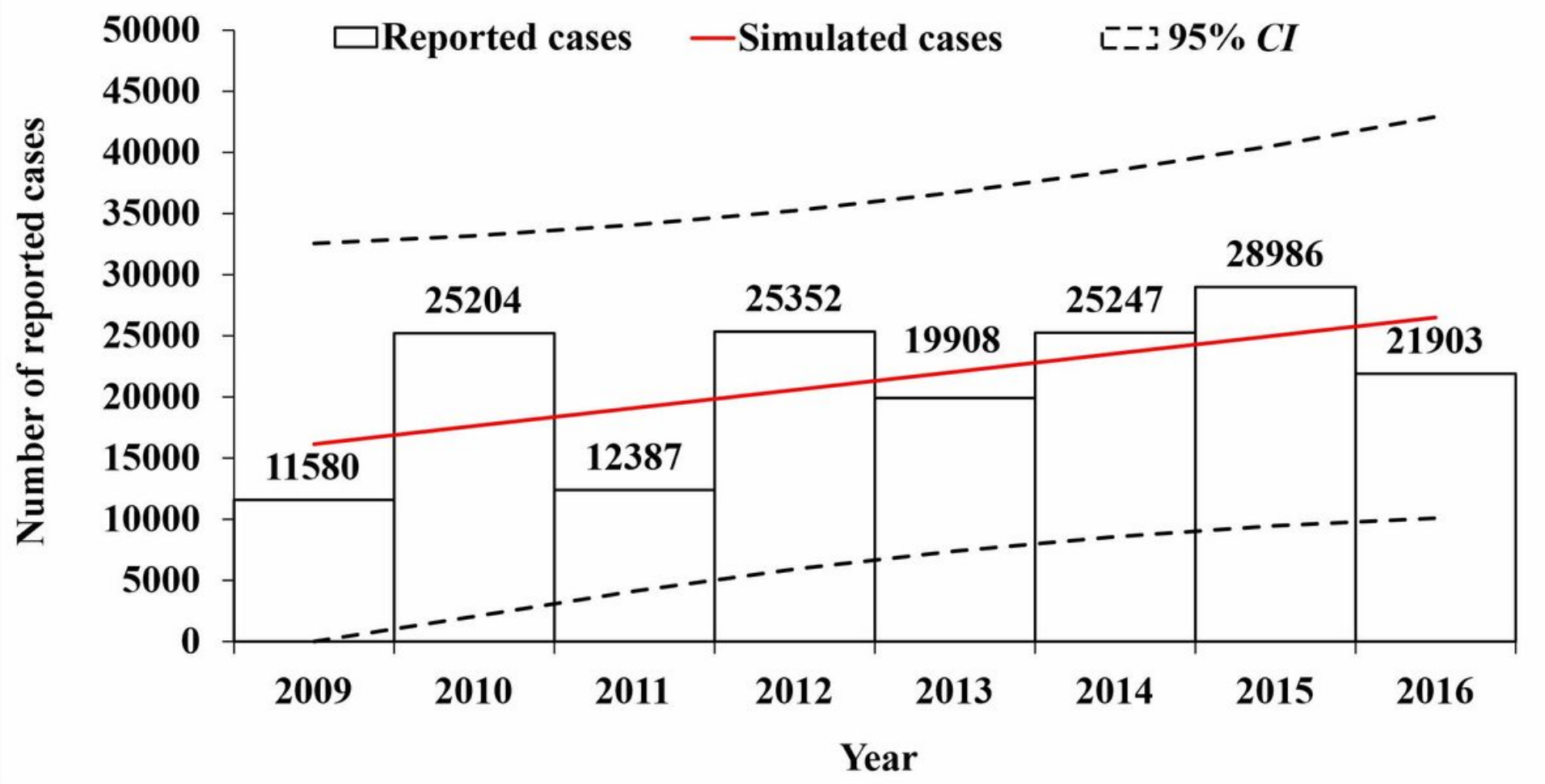

Figure 2

Yearly number of reported HFMD cases and its trend in Xi'an City.

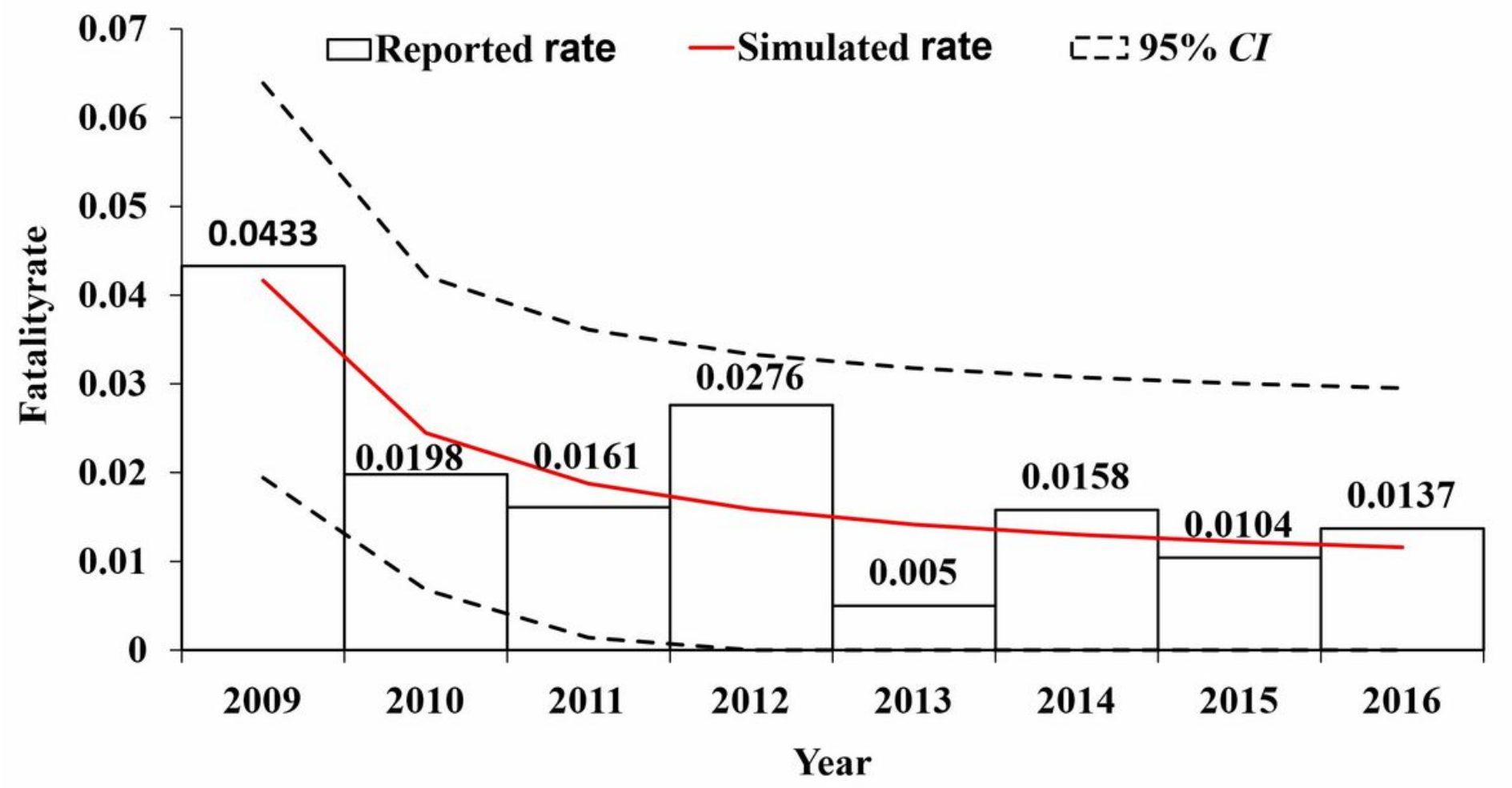

Figure 3 
Fatality of HFMD and its trend in Xi'an City.

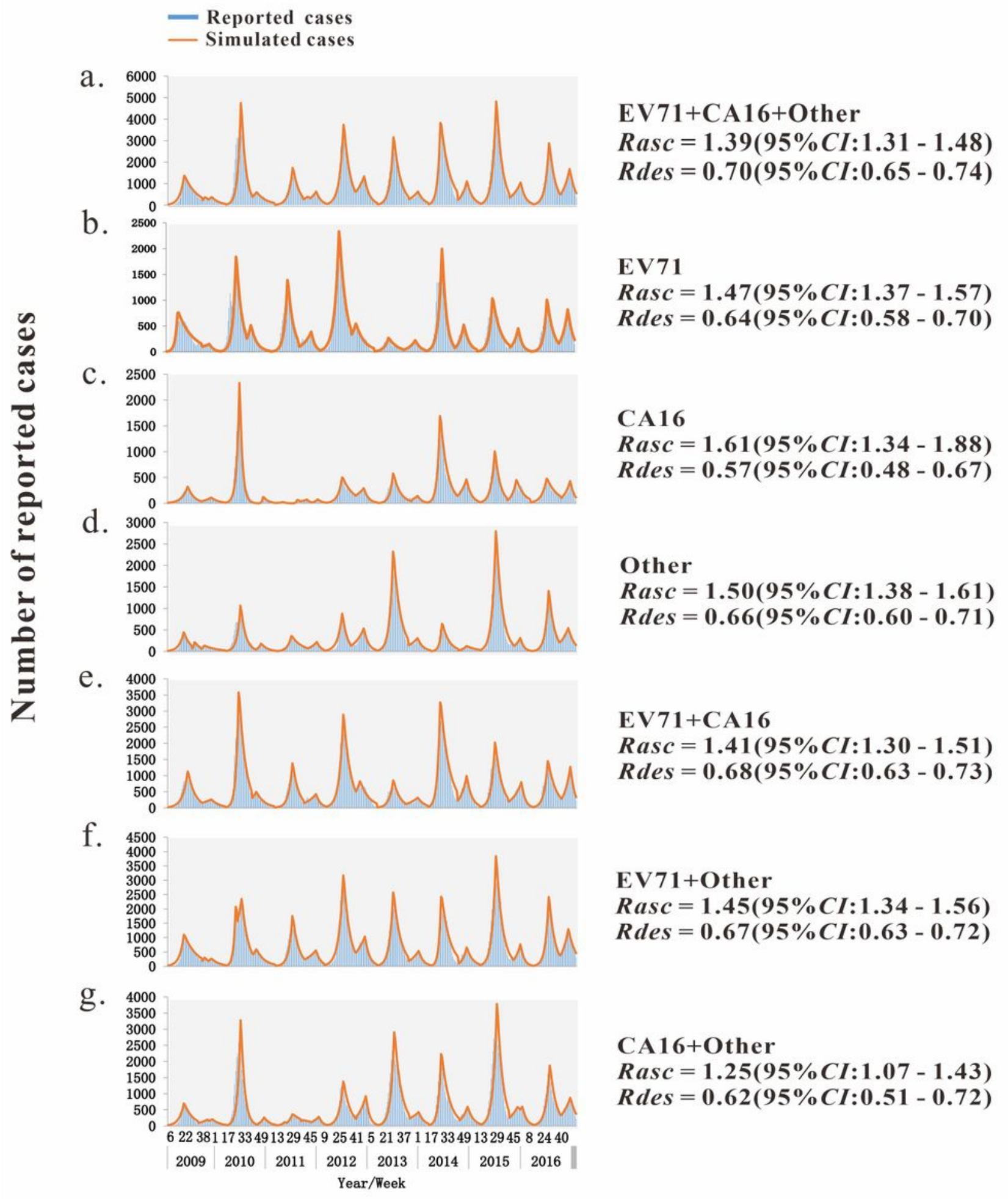

Figure 4

Results of model fitting and the transmissibility of HFMD's three pathogens. a: EV71+CA16+Other; $b$ : EV71: c, CA16; d: Other; e: EV71+CA16; f: EV71 +Other; g: CA16+Other. 
a: $R_{a s c}$
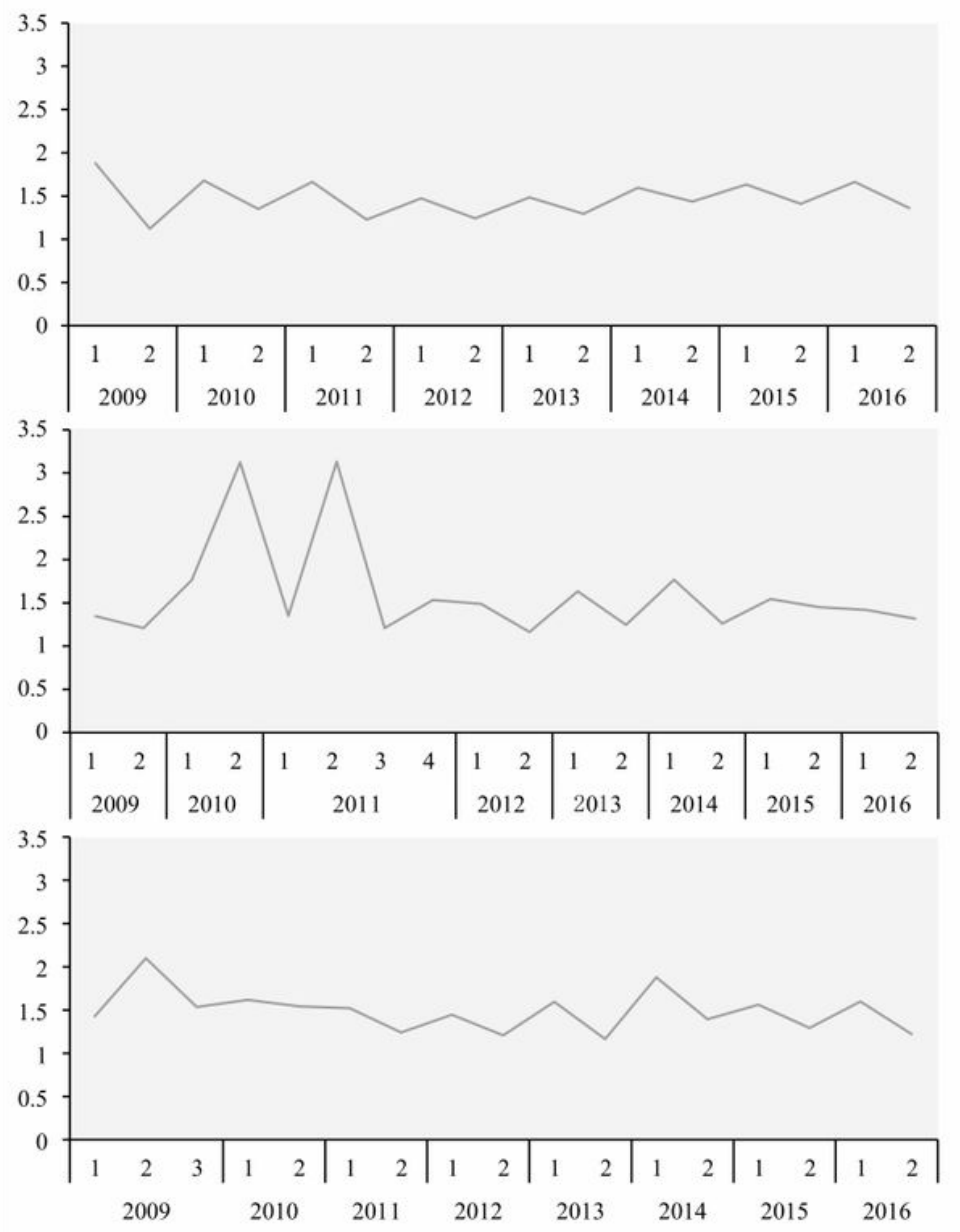

$\mathrm{b}: R_{\text {des }}$
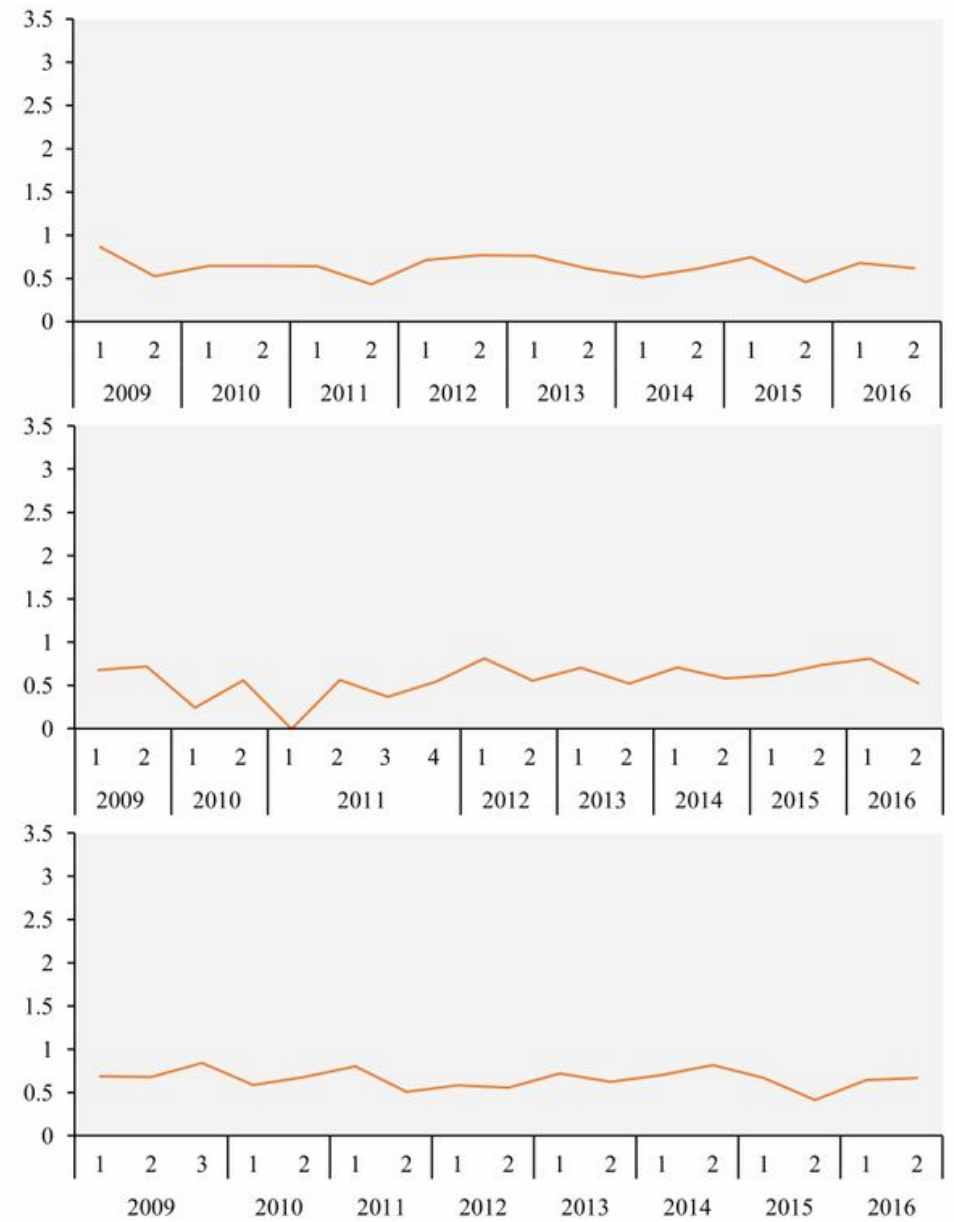

Figure 5

The trends of the transmissibility of HFMD. a: Rasc; b: Rdes. 
a: $R_{a s c}$

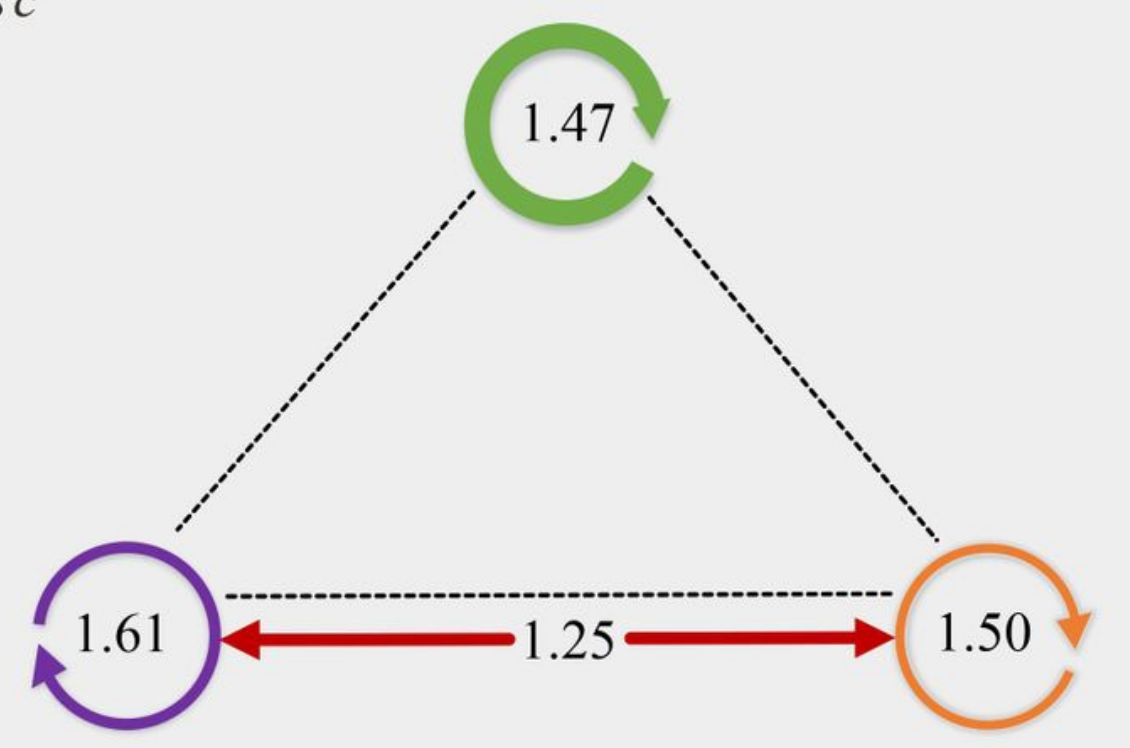

C EV71

CA 16

Other

$\rightarrow$ Interaction

$\mathrm{b}: R_{\text {des }}$

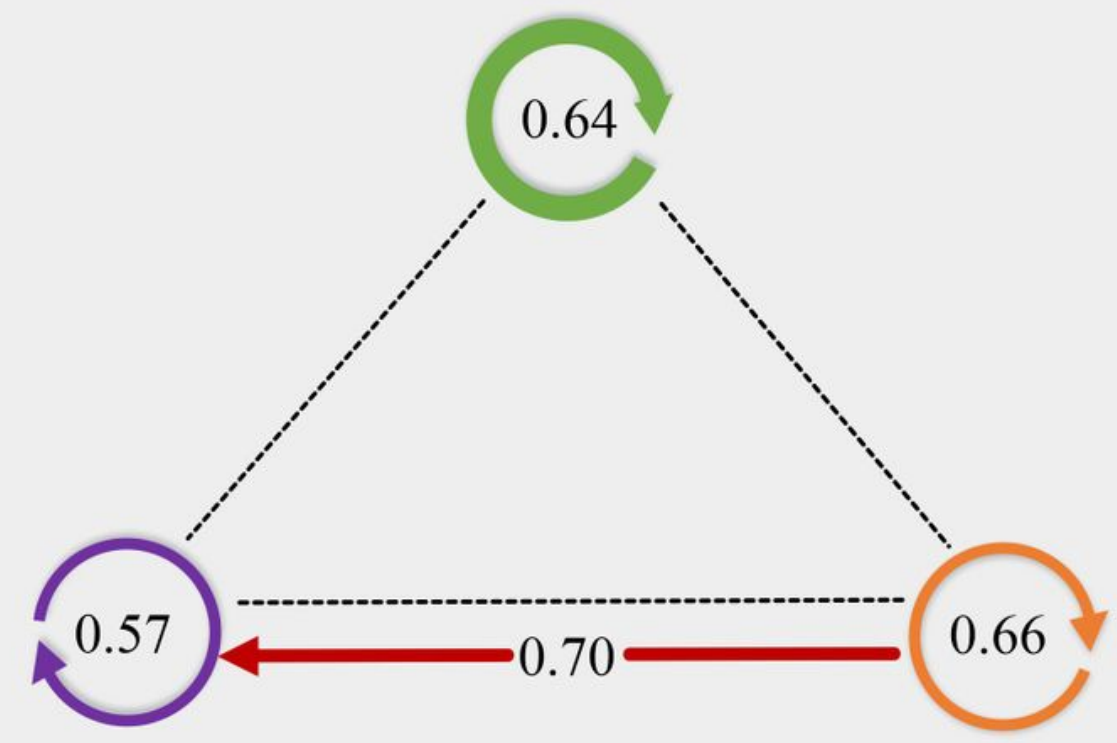

Figure 6

The interaction modes of the transmissibility of HFMD. a: Rasc; b: Rdes; Arrow indicates the direction of interaction from one pathogen to another one; no arrow refers to no interaction.

\section{Supplementary Files}


This is a list of supplementary files associated with this preprint. Click to download.

- Methodsformulas.docx

- Table1.doc 